\begin{tabular}{|c|l|}
\hline Title & Cytochemical and cytological properties of perineuronal oligodendrocytes in the mouse cortex \\
\hline Author(s) & $\begin{array}{l}\text { Takasaki, Chihiro; Y amasaki, Miwako; Uchigashima, Motokazu; Konno, Kohtarou; Y anagawa, Y uchio; W atanabe, } \\
\text { Masahiko }\end{array}$ \\
\hline Citation & $\begin{array}{l}\text { European Journal of Neuroscience, 32(8), 1326-1336 } \\
\text { https://doi.org/10.1111J.1460-9568.2010.07377.x }\end{array}$ \\
\hline Issue Date & 2010-10 \\
\hline Doc URL & http://hdl.handle.net/2115/47190 \\
\hline Rights & The definitive version is available at wileyonlinelibrary.com \\
\hline Type & article (author version) \\
\hline File Information & EJN32-8_1326-1336.pdf \\
\hline
\end{tabular}

Instructions for use 


\title{
Cytochemical and cytological properties of perineuronal oligodendrocytes in the mouse cortex
}

\author{
Chihiro Takasaki ${ }^{1,2}$, Miwako Yamasaki ${ }^{1}$, Motokazu Uchigashima ${ }^{1}$, Kohtarou \\ Konno $^{1}$, Yuchio Yanagawa ${ }^{3,4}$, Masahiko Watanabe ${ }^{1,4}$
}

\begin{abstract}
${ }^{1}$ Department of Anatomy, Hokkaido University Graduate School of Medicine, Sapporo 060-8638, ${ }^{2}$ Department of Pediatric Dentistry, Hokkaido University Graduate School of Dentistry, Sapporo 060-8586, ${ }^{3}$ Department of Genetic and Behavioral Neuroscience, Gunma University Graduate School of Medicine, Maebashi 371-8511, ${ }^{4}$ Japan Science and Technology Agency, CREST, Tokyo 102-0075, Japan
\end{abstract}

Running Title: perineuronal oligodendrocytes in mouse cortex

Abstract, 246 words; Introduction, 422 words.

7 Figures with 2 Supplemental Figures

Correspondence and proofs to: Masahiko Watanabe

Department of Anatomy, Hokkaido University School of Medicine, Sapporo 060-8638, Japan. TEL, +81-11-706-5032; FAX, +81-11-706-5031

E-mail, watamasa@med.hokudai.ac.jp 


\section{ABSTRACT}

Neuronal cell bodies are associated with glial cells collectively referred to as perineuronal satellite cells. One such satellite cell is the perineuronal oligodendrocyte, which is unmyelinating oligodendrocytes attaching to large neurons in various neural regions. However, little is known about their cellular characteristics and function. In this study, we identified perineuronal oligodendrocytes a's $\mathbf{Z}^{\prime}$-cyclic nucleotide 3'-phosphodiesterase (CNP)-positive cells attaching to neuronal perikarya immunostained for microtubule-associated protein 2, and examined their cytochemical and cytological properties in the mouse cerebral cortex. CNP-positive perineuronal oligodendrocytes were immunonegative to representative glial markers for astrocytes (brain-type lipid binding protein and glial fibrillary acidic protein), microglia (Iba-1), and $\mathrm{NG}^{+}$glia (NG2). Instead, almost all perineuronal oligodendrocytes expressed glia-specific or glia-enriched metabolic enzymes, i.e., creatine synthetic enzyme S-adenosylmethionine:guanidinoacetate $\mathrm{N}$-methyltransferase and L-serine biosynthetic enzyme 3-phosphoglycerate dehydrogenase. As to molecules participating in the glutamate-glutamine cycle, none of perineuronal oligodendrocytes expressed plasmalemmal glutamate transporters GLAST and GLT-1, although nearly a half of perineuronal oligodendrocytes were immunopositive for glutamine synthetase. Cytologically, perineuronal oligodendrocytes were mainly distributed in deep cortical layers (layers IV-VI), and attached directly and tightly to neuronal cell bodies, making a long concaved impression to the contacting neurons. Interestingly, they attached more to glutamatergic principal neurons than to GABAergic interneurons, and this became evident at postnatal day 14, when the cerebral cortex develops and maturates. These cytochemical and cytological properties suggest that perineuronal oligodendrocytes are so differentiated as to fulfill metabolic support to the associating principal cortical neurons, rather than to regulate their synaptic transmission. 


\section{INTRODUCTION}

Almost entire surface of neurons, except for synaptic contact sites, is covered by elements of various glial cells (Hyden, 1961). Glial cells attaching or surrounding neuronal cell bodies are referred to as perineuronal satellite cells, and comprise oligodendrocytes, astrocytes, and microglia (Brownson, 1960; Ogawa et al., 1985). In the cerebral cortex, the percentage of neurons associated with perineuronal satellite cells increases with age, and the highest population of perineuronal satellites is derived from the oligodendrocyte (Brownson, 1960).

From the initial description by Del Rio-Hortega (1921), oligodendrocytes have been subdivided into the interfascicular and perineuronal types, of which the former is myelinating cells distributed mainly in the white matter (Peters, 1964; Bunge, 1968). On the other hand, perineuronal oligodendrocytes mainly reside in the gray matter, and appose directly to neuronal cell bodies without forming any morphological specialization between them (Peters et al., 1976). They are assumed to be unmyelinating cells in normal brains, but are capable of producing myelin when remyelination of axons is induced after demyelination (Ludwin, 1978, 1979, 1984). Like astrocytes, perineuronal oligodendrocytes, but not white matter oligodendrocytes, express glutamine synthetase, a converting enzyme of glutamate into glutamine (Cammer, 1990; D’Amelio et al., 1990). In addition, perineuronal oligodendrocytes protect against neuronal apoptosis through remarkable up-regulation of lipocalin-type prostaglandin D synthase (L-PDGS; Taniike et al., 2002). These findings point to their supportive nature for associating neurons under physiological and pathophysiological conditions. Furthermore, association of perineuronal oligodendrocytes with particular types of cortical neurons is inferred from the fact that perineuronal oligodendrocytes, which are distributed in the inner third of the cerebral cortex in control mice, spread over the entire width in the reeler mouse, a spontaneous mutant with malpositioning of cortical neurons 
(LeVine and Torres, 1993). However, little is known to date about their cytochemical properties and cytological characteristics.

In the present study, we undertook this issue by identifying perineuronal oligodendrocytes as 2',3'-cyclicnucleotide 3'-phosphodiesterase (CNP)-positive cells that attach to neuronal somata expressing microtubule-associated protein-2 (MAP2). We found in the mouse cerebral cortex that perineuronal oligodendrocytes were rich in glia-specific or glia-enriched metabolic enzymes, including synthetic enzymes for creatine, L-serine, and glutamine. By contrast, they lacked glial plasmalemmal glutamate transporters, which, together with glutamine synthetase, build up the glutamate-glutamine cycle to regulate and maintain synaptic transmission. Nevertheless, they attached more to glutamatergic principal neurons than to GABAergic interneurons, and their tight attachment became evident by the end of the second postnatal week. Therefore, it appears likely that perineuronal oligodendrocytes are so differentiated in the cerebral cortex as to meet increasing demands of principal neurons for metabolic support.

\section{MATERIAL AND METHODS}

Animal and section preparation. We used C57BL/6J mice at postnatal day 0 (P0)-P21 and the adult stage (2-4 months of age). We also used glutamate decarboxylase 67 (GAD67)-green fluorescent protein (GFP) (Aneo) mice (Tamamaki et al., 2003), which were termed GAD67-GFP knock-in mice in the present study. The day of birth ( 24 hr) was designated as P0. Mice were treated according to the guidelines for the care and use of laboratory animals of the Hokkaido University Graduate School of Medicine. Under deep pentobarbital anesthesia (100 mg/kg of body weight, i.p.), mice were fixed by transcardial perfusion with $4 \%$ paraformaldehyde/ $0.1 \%$ picric acid in $0.1 \mathrm{M}$ sodium 
phosphate buffer ( $\mathrm{pH}$ 7.2, PB) for immunofluorescence microscopy and 4\% paraformaldehyde/0.1\% glutaraldehyde in $0.1 \mathrm{M}$ PB for immunoelectron microscopy. Sections for immunohistochemical analyses were prepared using a microslicer (40 $\mu \mathrm{m}$ in thickness; VT1000S; Leica Microsystems, Nussloch, Germany) or cryostat (30 $\mu \mathrm{m}$; CM1900; Leica). The somatosensory cortex was analyzed in the present study.

Antibody. The nucleotide sequence encoding mouse glutamine synthetase (GS) (amino acid residues 331-373, GenBank Accession No. X16314), Neisseria gonorrhoeae GFP (full sequence, YP_002302326), or mouse GAT1 (1-44 and 564-599, NM_178703) was amplified by polymerase chain reaction, and subcloned into the pGEX4T-2 plasmid vector (Amersham Biosciences, Bucks, UK) to be expressed as glutathione S-transferase (GST) fusion protein. Fusion proteins were emulsified with Freund's complete adjuvant in the first immunization and incomplete adjuvant in the subsequent immunization (DIFCO, Detroit, MI, USA), and injected subcutaneously into female guinea pigs and rabbits at intervals of 2 weeks. Two weeks after the six injection, immunogen-specific antibody was collected by affinity purification using GST-free polypeptides coupled to CNBr-activated Sepharose 4B (Amersham Biosciences). GST-free peptides were prepared by in-column thrombin digestion of GST fusion proteins bound to glutathione-Sepharose 4B media. The specificity of GS antibody was tested by immunoblot and preabsorption test (Fig. 1), while that of GFP antibody was by specific neuronal labeling in GAD67-GFP knock-in mice, but not in control mice (data not shown). The specificity of GAT1 antibodies was confirmed by similar labeling patterns with use of two antibodies against the $N$ - and $C$-terminal regions (Supplemental Fig. S1): immunoblot detection of protein band at $65 \mathrm{kDa}$, similar overall immunolabeling in the brain, and intense labeling of GABAergic axons and terminals, as reported previously (Minelli et al., 1995; Jursky and Nelson, 1996). 
We also used goat microtubule-associated protein 2 antibody (MAP2, $1 \mu \mathrm{g} / \mathrm{ml}$, Miura et al., 2006), mouse CNP antibody (diluted to 1:600; C5922, Sigma, St. Louis, MO), guinea pig $S$-adenosylmethionine:guanidinoacetate $N$-methyltransferase (GAMT) antibody (1 $\mu \mathrm{g} / \mathrm{ml}$, Tachikawa et al., 2004), rabbit and guinea pig 3-phosphoglycerol dehydrogenase (Phgdh) antibodies (1 $\mu \mathrm{g} / \mathrm{ml}$, Yamasaki et al., 2001), rabbit and guinea pig glutamate transporter GLAST antibodies (1 $\mu \mathrm{g} / \mathrm{ml}$, Shibata et al., 1997), rabbit GLT-1 antibody (1 $\mu \mathrm{g} / \mathrm{ml}$, Yamada et al., 1998), rabbit brain-type lipid binding protein (BLBP, $1 \mu \mathrm{g} / \mathrm{ml}$, Yamada et al., 2000), rabbit glial fibrillary acidic protein antibody (GFAP, $1 \mu \mathrm{g} / \mathrm{ml}$, Hisano et al., 2009), rabbit and guinea pig vesicular inhibitory amino acid transporter (VIAAT1, $\mu \mathrm{g} / \mathrm{ml}$, Fukudome et al., 2004)), rabbit NG2 antibody (1:200; AB5320, CHEMICON International, Temecula, CA, USA), and rabbit Iba-1 antibody (1:500, Wako Pure Chemical Industries, Japan).

Immunohistochemistry. All incubations were performed at room temperature. Following overnight incubation with primary antibodies, sections were incubated with Alexa488-, Cy3-, or Cy5-conjugated species-specific secondary antibodies for $2 \mathrm{hr}$ (Invitrogen, Carlsbad, CA; Jackson ImmunoResearch, West Grove, PA). For fluorescent Nissl staining, some sections were incubated with NeuroTrace 500/525 Green (NTG, Molecular Probes, Eugene, OR) or TOTO3 (Invitrogen) for 15 min. Images were taken with a laser scanning microscopy (FV1000, Olympus, Tokyo, Japan) equipped with HeNe/Ar laser, and PlanApo (10x/0.40) and PlanApoN (60x/1.42, oil immersion) objective lens (Olympus). To avoid cross-talk between multiple fluorophores, Alexa 488 (or FITC), Cy3, and Cy5 (or TOTO3) fluorescent signals were acquired sequentially using the 488-nm, 543-nm, and $633 \mathrm{~nm}$ excitation laser lines. Whole-section mosaics of low-magnification images were assembled using a MetaMorph software (Molecular Devices, Downingtown, PA). For high-magnification images, single optical sections 
were obtained $(640 \times 640$ pixels, pixel size $110 \mathrm{~nm})$ for presentation.

In pre-embedding silver-enhanced immunogold staining, microslicer sections were dipped in 5\% bovine serum albumin (BSA)/0.02\% saponin/phosphate-buffered saline (PBS) for 30 min and incubated overnight with GAMT antibody diluted with 1\% BSA/0.004\% saponin/PBS followed by anti-guinea pig IgG linked to 1.4-nm gold particles (Nanogold; Nanoprobes Inc., Stony Brook, NY, USA) for 2 hr. Immunogold staining was intensified with a silver enhancement kit (HQ silver, Nanoprobes). Then, sections were treated with $1 \%$ osmium tetroxide for 15 min, stained in blocks with $2 \%$ uranyl acetate for 20 min, dehydrated, and embedded in Epon 812. Photographs were taken with an H-7100 electron microscope (Hitachi, Tokyo, Japan).

Immunoblot. Homogenate of the adult mouse cerebral cortex was prepared by sonication in ice-cold buffer containing 10 mM Tris-HCl (pH 7.0), 1 mM EDTA, 1 mM EGTA and $0.32 \mathrm{M}$ sucrose. Protein concentration was determined by the Lowry's method. Protein samples (5, 10, or $20 \mu \mathrm{g}$ ) were separated by $7.5 \%$ sodium dodecylsulfate polyacrylamide gel electrophoresis under reduced conditions, and electroblotted onto a nitrocellulose membrane (Schleicher \& Schuell, Dassel, Germany). The membrane was immunoreacted with primary antibodies ( $1 \mu \mathrm{g} / \mathrm{ml}$ for each) for $1 \mathrm{hr}$, and immunoreactions were visualized using the ECL detection system (Amersham, Ducks, UK).

Fluorescent in situ hybridization (FISH). Procedures for FISH were reported previously (Yamasaki et al., 2010). In brief, fresh frozen sections were hybridized with digoxigenin (DIG)- or fluorescein-labeled cRNA probes for mRNAs of mouse CNP (nucleotide residues 2101-3044, NM_001146318.1), proteolipid protein (PLP, 1-1359, NM_011123.2), GLT1 (595-1563, NM_001077514.3), GLAST (1620-2576, 
BC066154.1), or L-PGDS (337-730, BC038083). In double FISH, DIG and fluorescein were detected by the two-step method: the first detection with peroxidase-conjugated anti-fluorescein antibody (Roche Diagnostics, 1:500, $1 \mathrm{hr}$ ) and the FITC-TSA plus amplification kit (PerkinElmer, Shelton, CT), and the second detection with peroxidase-conjugated anti-DIG antibody (Roche Diagnostics, 1:500, $1 \mathrm{hr}$ ) and the Cy5-TSA plus amplification kit (PerkinElmer). Residual activities of peroxidase introduced in the first detection were inactivated by incubation of sections with $0.6 \%$ $\mathrm{H}_{2} \mathrm{O}_{2}$ for 30 min. When combining FISH with immunofluorescence, FISH preceded immunofluorescence. TOTO3 or NeuroTrace 530/615 Red (NTR, Invitrogen) was used for fluorescent counterstaining. The specificity of FISH was tested by blank labeling with use of control sense cRNA probes.

\section{RESULTS}

Identification of perineuronal oligodendrocytes

CNP is one of the glial markers specific to oligodendrocytes. To visualize oligodendrocytes, we used mouse monoclonal CNP antibody, which specifically recognized a single protein band at $45 \mathrm{kDa}$ in the adult mouse brain (Fig. 1A), as reported previously (Sprinkle et al., 1987). The specificity of CNP immunohistochemistry was assessed by comparing with FISH labeling patterns for CNP mRNA (Fig. 2). Cells expressing CNP mRNA were distributed densely in the white matter and relatively sparsely in the gray matter (Fig. 2A1). The specificity of FISH for CNP mRNA was shown by almost complete overlap with PLP mRNA, another marker gene specific to oligodendrocytes (Fig. 2A-C). In the cerebral cortex and other brain regions, cellular populations immunolabeled with CNP antibody matched completely with those expressing CNP mRNA (Fig. 2D, E). These results indicate the specificity of 
immunohistochemical signals with use of this CNP antibody.

When applying the CNP antibody together with fluorescent counterstains, CNP-immunoreactive elements were distributed in the adult cerebral cortex in a deep-to-superficial gradient (Fig. 2D, 3A). At high magnifications, CNP was detected in perikarya of small oval cells and in processes traversing the neuropil (Fig. 2E). When double immunostained for MAP2, a marker for neuronal perikarya and dendritic shafts, many CNP-positive cells were found to attach to MAP2-labeled large neuronal perikarya (Fig. 3B). They were not labeled for other glial markers, including BLBP and GFAP for astrocytes ( 0 of 61 or 60 CNP-positive cells, respectively), Iba-1 for microglia (0 out of 62 CNP-positive cells), and NG2 for $\mathrm{NG}^{+}$glia (0 out of 61 CNP-positive cells) (Fig. 3C-F). Instead, most cells expressing CNP mRNA in the cerebral cortex also expressed L-PDGS mRNA (60 out of 68 cells), and all cells expressing L-PGDS mRNA-positive cells expressed CNP mRNA (60 out of 60 cells) (Fig. 2G, H). L-PDGS is a major synthetic enzyme of prostaglandin $D_{2}$, being expressed in leptomeninges, choroid plexus, and oligodendrocytes (Urade et al., 1993) and upregulated in perieneuronal oligodendrocytes in a demyelinating animal model (Taniike et al., 2002). These results suggest that CNP can be used as a selective and useful marker to visualize perieneuronal oligodendrocytes.

In the following analyses, we identified perineuronal oligodendrocytes as CNP-positive satellites attaching to MAP2-positive neurons, unless otherwise noted.

\section{Perineuronal oligodendrocyte highly expresses glial metabolic enzymes}

Their cytochemical properties were further characterized by immunofluorescence for glia-specific or glia-enriched metabolic enzymes and transporters, including creatine synthetic enzyme GAMT, L-serine synthetic enzyme Phgdh, glutamine synthetic enzyme GS, and plasmalemmal glutamate transporter GLAST. The specificity of 
antibodies against these molecules was tested by immunoblot using cortical extracts (Fig. 1A). Each antibody recognized single protein bands at positions expected from deduced molecular masses: GAMT at $27 \mathrm{kDa}$, Phgdh at $57 \mathrm{kDa}$, GS at $43 \mathrm{kDa}$, and GLAST at 65kDa. As shown below (Fig. 4), immunohistochemistry with use of these antibodies yielded selective or preferential glial labeling, which made neuronal cell bodies as dark silhouettes. The apparent sizes of immunoblot bands and exclusive glial labeling were consistent with previous reports (Hallermayer and Hamprecht 1984; Shibata et al., 1997; Yamasaki et. al., 2001; Tachikawa et al., 2004). Therefore, immunohistochemical labeling with use of these primary antibodies was judged to be specific.

By triple immunofluorescence, perikarya of CNP-positive perineuronal oligodendrocytes showed intense signals for GAMT (arrow, Fig. 4A) and Phgdh (arrow, Fig. 4B). By cellular counting, GAMT and Phgdh were detected in 100\% (61 out of 61 cells) or 98\% (59 out of 60), respectively, of CNP-positive perineuronal oligodendrocytes. When identifying perineuronal oligodendrocytes by GAMT immunofluorescence, CNP was detected in 95\% (61 out of 64) of GAMT-positive perineuronal oligodendrocytes, indicating that most perineuronal oligodendrocytes coexpress CNP and GAMT. Among perineuronal oligodendrocytes, there were GS-positive (arrow, Fig. 4C) and GS-negative (arrowhead, Fig. 4D) populations. The percentage of GS-expressing perineuronal oligodendrocytes was 57\% (36 out of 63) of CNP-positive perineuronal oligodendrocytes.

In contrast, GLAST was detected in none of perineuronal oligodendrocytes (arrowhead, 0 out of 60, Fig. 4E). This was also true for another glial glutamate transporter GLT-1 (arrowhead, 0 out of 62 cells, Fig. 4F). The lack of glial glutamate transporter expression was further ascertained by negative CNP immunolabeling in processes of perineuronal oligodendrocytes (Fig. 5A for GLT1; data not shown for 
GLAST) and also by negative FISH labeling for GLT1 (Fig. 5B, C, F) and GLAST (Fig. 5D, E, G) mRNAs in oligodendrocytes expressing CNP mRNA. Therefore, perineuronal oligodendrocytes are rich in enzymes mediating neuron-glial metabolic interplay, whereas they lack glial glutamate transporters regulating synaptic transmission.

\section{Cytological properties of perineuronal oligodendrocyte}

The distribution of perineuronal oligodendrocytes in the cerebral cortex was assessed by triple fluorescent labeling for CNP, MAP2, and fluorescent Nissl stain NTG. Because results from two mice were similar, scores were pooled together. CNP-positive perineuronal oligodendrocytes attaching to MAP2-positive/NTG-positive neuronal cell bodies constituted 44\% of the total number of CNP-positive oligodendrocytes (151 out of 341 cells), although the score should be underestimated due to their identification using single optical sections by confocal laser scanning microscopy. These perineuronal oligodendrocytes were mainly distributed in deep cortical layers: no cell in the layer I (0\%), 6 cells in the layer II/III (4\%), 36 cells in the layer IV (24\%), 58 cells in the layer V (38\%), and 51 cells in the layer VI (34\%).

Then, ultrastructural features of perineuronal oligodendrocytes were examined by silver enhanced-immunoelectron microscopy using GAMT antibody, rather than CNP antibody, because GAMT antibody yielded preferential labeling in perineuronal oligodendrocytes: 5.9 metal particles per $1 \mu \mathrm{m}^{2}$ of perikarya of perineuronal oligodendrocytes (measured area, $13.1 \mu^{2}$ ) and 0.8 particles per $1 \mu \mathrm{m}^{2}$ of perikarya of the attaching neurons $\left(45.2 \mu \mathrm{m}^{2}\right)$. Metal particles for GAMT were deposited in the cytoplasm of oval or polygonal cells, which had round a nucleus rich in dark heterochromatin (Fig. 6A). Consistent with previous description (Peters et al., 1976), these perineuronal oligodendrocytes attached directly to neurons having a large pale nucleus, and excluded other cellular elements from their contact sites (Fig. 6A-D). Tight 
attachment was appreciated from a long curved or concaved impression made on to the contacting neurons (Fig. 6A) and from parallel alignment of two plasma membranes with a narrow intercellular space (Fig. 6C).

\section{Perineuronal oligodendrocytes differentiate with cerebral cortex maturation}

The ontogeny of phenotypic differentiation as perineuronal oligodendrocytes was followed from birth to P21. Because perikaryal expression of CNP and MAP2 was very low in neonatal cortex, we used GAMT immunofluorescence instead of CNP to label perineuronal oligodendrocytes, and also used NTG together with MAP2 immunofluorescence to label neuronal perikarya (Fig. 7A-D). GAMT-positive cells were already dispersed in the cortex at P1 and P7, but they appeared to touch NTG/MAP2-labeled neurons only lightly (Fig. 7A, B). At P14 and P21, GAMT-positive cells often made characteristic curved impression on to NTG/MAP2-labeled neurons, as observed in the adult stage (Fig. 4, 6). Therefore, cytological differentiation of perineuronal satellite cells likely proceeds with postnatal maturation of the cerebral cortex.

\section{Perineuronal oligodendrocytes preferentially associate glutamatergic neurons}

Glutamatergic neurons are the principal neurons constituting about two thirds of cortical neurons, while the rest are GABAergic interneurons (Sloper et al., 1979; Gabbott and Somogyi, 1986). Finally, we asked which types of cortical neurons were associated with perineuronal oligodendrocytes. To this end, we applied triple immunofluorescence for CNP, MAP2, and GFP to GAD67-GFP knock-in mice at P14 and adult stage, in order to distinguish GFP-positive GABAergic neurons from GFP-negative glutamatergic ones (Fig. 7E-H). Because results from two mice yielded similar scores at each stage, data from two mice were pooled together. 
By random sampling of neuronal images from single optical sections, CNP-positive perineuronal oligodendrocytes often attached to GFP-negative/MAP2-positive glutamatergic neurons (Fig. 7E, G). The percentage of the glutamatergic neurons directly attaching to perineuronal oligodendrocytes was 2.8\% (60 out of 2123 glutamatergic cells) at P14 and 3.6\% (119 out of 3265 glutamatergic cells) at the adult stage. In contrast, the percentage of GFP-positive GABAergic neurons contacted by perineuronal oligodendrocytes was as low as $0.27 \%$ ( 1 out of 374 GABAergic cells) at P14 and $0.80 \%$ (5 out of 627 GABAergic cells) at the adult stage (Fig. 7F, H). Thus, perineuronal oligodendrocytes are satellite cells preferential for glutamatergic, rather than GABAergic, cortical neurons.

\section{DISCUSSION}

In the present study, we analyzed cytochemical and cytological properties of perineuronal oligodendrocytes in the mouse cerebral cortex. We found that almost all perineuronal oligodendrocytes expressed Phgdh and GAMT. Phgdh is an initial step enzyme of biosynthetic pathway for L-serine, which serves as a building block of proteins and is also utilized for synthesis of various biomolecules, including glycine, L-cysteine, phosphatidylserine, ceramide, and D-serine (Ichihara and Greenberg, 1957; Snell, 1984). Glycine is further used for creatine biosynthesis through two sequential steps catalyzed by L-arginine:glycine amidinotransferase and GAMT. The creatine/phosphocreatine shuttle system is essential for the storing and buffering of high phosphate-bound energy in tissues with high and fluctuating energy demands, such as skeletal muscles and the brain (Wyss and Kaddurah-Daouk, 2000). Both Phgdh and GAMT are exclusively expressed in astrocytes and oligodendrocytes in the adult brain (Furuya et al., 2000; Yamasaki et al., 2001; Sakai et al., 2003; Yamashita et al., 2003; 
Tachikawa et al., 2004). Importantly, exogenous supply of glycine or L-serine greatly promotes the survival and differentiation of cultured neurons (Furuya et al., 2000). Taking these into consideration, the expression of Phgdh and GAMT in perineuronal oligodendrocytes suggests that they also support neuronal survival, differentiation, and function, as has been proposed for astrocytes and white matter oligodendrocytes (Du and Dreyfus, 2002; Furuya and Watanabe, 2003). Intriguing with this point, the number of perineuronal oligodendrocytes is reduced prominently and significantly in the prefrontal cortex of patients with schizophrenia and mood disorders (Vostrikov et al., 2007). Furthermore, deficiency in Phgdh or GAMT in humans leads to severe neurological symptoms, including severe mental retardation and epilepsy (de Koning et al., 1998; Schulze, 2003; Lion-François et al., 2006). Therefore, it seems interesting to address in future studies the role of Phgdh and GAMT in perineuronal oligodendrocytes in relation with disorders of cortical function and development.

By contrast, none of perineuronal oligodendrocytes expressed glial glutamate transporters GLAST and GLT-1. These plasmalemmal transporters play a critical role in keeping the extracellular glutamate concentration low enough to terminate glutamate receptor activation and to protect neurons from glutamate excitotoxicity (Hertz, 1979; Choi, 1992). Moreover, these transporters constitute, in cooperation with GS, the glutamate-glutamine cycle. Glutamate released from presynaptic terminals is rapidly taken up by GLAST and GLT-1 on astrocytes, and converted to glutamine by GS in astrocytes. This conversion contributes to maintain glutamate uptake function by astrocytes and to supply a precursor of glutamate to presynaptic terminals as a non-toxic form of glutamine (Tsacopoulos and Magestretti, 1996; Bacci et al., 1999; Haydon, 2001). The lack of GLAST and GLT-1 indicates that perineuronal oligodendrocytes are involved little, if any, in the glutamate-glutamine cycle. Rather, expression of GS in nearly a half of perineuronal oligodendrocytes might reflect its metabolic role to 
maintain the concentration of neurotoxic ammonia low by incorporating it into glutamine (Cooper and Plum, 1987). Taking the distinct cytochemical characteristics together with protective role against neuronal apoptosis through up-regulation of L-PGDS (Taniike et al., 2002), perineuronal oligodendrocytes are likely the satellite dedicated to fulfill metabolic support for their associating neurons rather than to regulate synaptic transmission. This notion is further supported by the lack in perineuronal oligodendrocytes of GAT1 (Supplemental Fig. S2), a plasmalemmal GABA transporter expressed in GABAergic neurons and astrocytes (Minelli et al., 1995).

Despite the lack of glutamate transporters, perineuronal oligodendrocytes in the adult cortex were attached to $3.8 \%$ of GAD67-GFP-negative (i.e., glutamatergic) neurons, which was 5 times more than those attached to GAD67-GFP-positive (GABAergic) neurons. This preferential attachment to glutamatergic neurons became evident at P14, when rodent's cerebral cortex undergoes drastic development and maturation (Bray and Mynlieff, 2009; Bandeira et al., 2009). Furthermore, perineuronal oligodendrocytes were enriched in deep cortical layers, where large to medium-sized neurons reside and project their axons to subcortical regions. Taken altogether, it can be assumed that perineuronal oligodendrocytes differentiate in the developing cerebral cortex to meet increasing demands of principal neurons for metabolic support, particularly those projecting to remote areas.

\section{ACKNOWLEDGEMENT}

This study was supported through Grant-in-Aid for Young Scientists (Start-up) (19890010 to CT), Grant-in-Aid for Young Scientists (B) (21792050 to CT), and Grant-in-Aid for JSPS Fellows (20-04030 to MU) provided by the Ministry of 
Education, Culture, Sports, Science and Technology of Japan. MU is a recipient of Research Fellowships of the Japan Society for the Promotion of Science for Young Scientists.

\section{ABBREVIATIONS}

BLBP, brain-type lipid binding protein

BSA, bovine serum albumin

CNP, 2', 3'-cyclic nucleotide 3'-phosphodiesterase

DIG, digoxigenin

FISH, fluorescent in situ hybridization

GAD67, glutamate decarboxylase 67

GAMT, S-adenosylmethionine:guanidinoacetate $N$-methyltransferase

GFAP, glial fibrillary acidic protein

GFP, green fluorescent protein

GS, glutamine synthetase

GST, glutathione $S$-transferase

L-PDGS, lipocalin-type prostaglandin D synthase

MAP2, microtubule-associated protein-2

NG2, NG2 ${ }^{+}$glia

NTG, NeuroTrace 500/525 Green

NTR, NeuroTrace 530/615 Red

P, postnatal day

$\mathrm{PB}$, phosphate buffer

PBS, phosphate-buffered saline

Phgdh, 3-phosphoglycerol dehydrogenase

PLP, proteolipid protein 
Takasaki et al (17)

VIAAT1, vesicular inhibitory amino acid transporter 


\section{REFERENCE}

Bacci, A., Verderio, C., Pravettoni, E., \& Matteoli, M. (1999) The role of glial cells in synaptic function. Philos. Trans. R. Soc. Lond. B Biol. Sci., 354, 403-409.

Bandeira, F., Lent, R., \& Herculano-Houzel, S. (2009) Changing numbers of neuronal and non-neuronal cells underlie postnatal brain growth in the rat. Proc. Natl. Acad. Sci. USA. 106, 14108-14113.

Bray, J.G. \& Mynlieff, M. (2009) Influx of calcium through L-type calcium channels in early postnatal regulation of chloride transporters in the rat hippocampus. Dev. Neurobiol., 69, 885-896.

Brownson, R.H. (1960) The effect of x-irradiation on the perineuronal satellite cells in the cortex of aging brains. J. Neuropathol. Exp. Neurol., 19, 407-414.

Bunge, R.P. (1968) Glial cells and the central myelin sheath. Physiol. Rev., 48, 197-251.

Cammer, W. (1990) Glutamine synthetase in the central nervous system is not confined to astrocytes. J. Neuroimmunol., 26,173-178.

Choi, D.W. (1992) Excitotoxic cell death. J. Neurobiol. 23, 1261-1276.

Cooper, A.J. \& Plum, F. (1987) Biochemistry and physiology of brain ammonia. Physiol. Rev., 67, 440-519.

D'Amelio, F., Eng, L.F. \& Gibbs, M.A. (1990) Glutamine synthetase immunoreactivity 
is present in oligodendoroglia of various regions of the central nervous system. Glia, $\mathbf{3}$, 335-341.

de Koning, T.J., Duran, M., Dorland, L., Gooskens, R., Van Schaftingen, E., Jaeken, J., Blau, N., Berger, R. \& Poll-The, B.T. (1998) Beneficial effects of L-serine and glycine in the management of seizures in 3-phosphoglycerate dehydrogenase deficiency. Ann. Neurol., 44, 261-265.

Del Rio-Hortega, P. (1921) Estudios sobre la neuroglia. La glia de escasas radiaciones (oligodendroglia). Bol. Real Soc. Espan. Hist. Nat. 21, 63-92.

Du, Y. \& Dreyfus, C.F. (2002) Oligodendrocytes as providers of growth factors. $J$. Neurosci. Res., 68, 647-654.

Fukudome, Y., Ohno-Shosaku, T., Matsui, M., Omori, Y., Fukaya, M., Taketo, M., Watanabe, M., Manabe, M. \& Kano, M. (2004) Two distinct classes of muscarinic action on hippoccampal inhibitory synapses: M2-mediated direct suppression and M1/M3-mediated indirect suppression through endocannabinoid signaling. Eur. J. Neurosci., 19, 2682-2692.

Furuya, S., Tabata, T., Mitoma, J., Yamada, K., Yamasaki, M., Makino, A., Yamamoto, T., Watanabe, M., Kano, M. \& Hirabayashi, Y. (2000) L-serine and glycine serve as major astroglia-derived trophic factors for cerebellar Purkinje neurons. Proc. Natl. Acad. Sci. USA., 97, 11528-11533.

Furuya, S. \& Watanabe, M. (2003) Novel neuroglial and glioglial relationships mediated 
by L-serine metabolism. Arch. Histol. Cytol., 66, 109-121.

Gabbott, P.L. \& Somogyi, P. (1986) Quantitative distribution of GABA-immunoreactive neurons in the visual cortex (area 17) of the cat. Exp. Brain. Res., 61, 323-331.

Hallermayer, K. \& Hamprecht, B. (1984) Cellular heterogeneity in primary cultures of brain cells revealed by immunocytochemical localization of glutamine synthetase. Brain Res., 295,1-11.

Haydon, P.G. (2001) GLIA: listening and talking to the synapse. Nat. Rev. Neurosci., 2, 185-193.

Hertz, L. (1979) Functional interactions between neurons and astrocytes I. Turnover and metabolism of putative amino acid transmitters. Prog. Neurobiol. 13, 277-323.

Hisano, K., Watanabe, M. \& Morimoto, Y (2009) Protective effects of the free radical scavenger edaravone against glutamate neurotoxicity in nearly pure neuronal culture. $J$. Anesth., 23, 363-369.

Hyden, H. (1961) Satellite cells in the nervous system. Sci. Am., 205, 62-70.

Ichihara, A. \& Greenberg, D.M. (1957) Further studies on the pathway of serine formation from carbohydrate. J. Biol. Chem., 224, 331-340.

Jursky, F. \& Nelson, N. (1996) Developmental expression of GABA transporters GAT1 
and GAT4 suggests involvement in brain maturation. J. Neurochem., 67, 857-867.

LeVine, S.M. \& Torres, M.V. (1993) Satellite oligodendrocytes and myelin are displaced in the cortex of the reeler mouse. Dev. Brain Res., 75, 279-284.

Lion-François, L., Cheillan, D., Pitelet, G., Acquaviva-Bourdain, C., Bussy, G., Cotton, F., Guibaud, L., Gérard, D., Rivier, C., Vianey-Saban, C., Jakobs, C., Salomons, G.S. \& des Portes, V. (2006) High frequency of creatine deficiency syndromes in patients with unexplained mental retardation. Neurology, 67, 1713-1714.

Ludwin, S.K. (1978) Central nervous system demyelinattion and remyelination in the mouse. An ultrastructural study of cuprizone toxicity. Lab. Invest., 39, 597-612.

Ludwin, S.K. (1979) The perineuronal satellite oligodendrocyte. A role in remyelination. Acta Neuropathol., 47, 49-53.

Ludwin, S.K. (1984) The function of perineuronal satellite oligodendrocyte: an immunohistochemical study. Neuropathol. Appl. Neurobiol., 10, 143-149.

Minelli, A., Brecha, N.C., Karschin, C., DeBiasi, S. \& Conti, F. (1995) GAT-1, a high-affinity GABA plasma membrane transporter, is localized to neurons and astroglia in the cerebral cortex. J. Neurosci., 15, 7734-7746.

Miura, E., Fukaya, M., Sato, T., Sugihara, K., Asano, M., Yoshioka, K. \& Watanabe, M. (2006) Expression and distribution of JNK/SAPK-associated scaffold protein JSAP1 in developing and adult mouse brain. J. Neurochem., 97, 1431-1446. 
Ogawa, Y., Edins, S. \& Wolff, J.R. (1985) Oligodendrocytes in the pons and middle cerebellar peduncle of the cat. Cell Tissue Res., 240, 541-552.

Peters, A. (1964) Further observations on the structure of myelin sheaths in the central nervous system. J. Cell Biol. 20, 281-296.

Peters, A., Palay, S.L. \& Webster, H. de F. (1976) The Fine Structure of the Nervous System. The Neurons and Supporting Cells. pp. 231-263. Philadelphia-London-Toronto, Saunders.

Sakai, K., Shimizu, H., Koike, T., Furuya, S., \& Watanabe, M. (2003) Neutral amino acid transporter ASCT1 is preferentially expressed in L-Ser-synthetic/storing glial cells in the mouse brain with transient expression in developing capillaries. J. Neurosci., 223, $550-560$.

Schulze, A. (2003) Creatine deficiency syndromes. Mol. Cell. Biochem., 244, 143-150.

Shibata, T., Yamada, K., Watanabe, M., Ikenaka, K., Wada, K., Tanaka, K. \& Inoue, Y. (1997) Glutamate transporter GLAST is expressed in the radial glia-astrocyte lineage of developing mouse spinal cord. J. Neurosci., 17, 9212-9219.

Sloper, J.J., Hiorns, R.W. \& Powell, T.P. (1979) A qualitative and quantitative electron microscopic study of the neurons in the primate motor and somatic sensory cortices. Philos. Tran.s R. Soc. Lond. B Bio.l Sci.. 285, 141-171. 
Snell, K. (1984) Enzymes of serine metabolism in normal, developing and neoplastic rat tissues. Adv. Enzyme Regul., 22, 325-400.

Sprinkle, T.J., Agee, J.F., Tippins, R.B., Chamberlain, C.R., Faguet, G.B. \& De Vries, G.H. (1987) Monoclonal antibody production to human and bovine 2':3'-cyclic nucleotide 3'-phosphodiesterase (CNPase): high-specificity recognition in whole brain acetone powders and conservation of sequence between CNP1 and CNP2.Brain Res., 426, 349-57.

Tachikawa, M., Fukaya, M., Terasaki, T., Ohtsuki, S. \& Watanabe, M. (2004) Distinct cellular expressions of creatine synthetic enzyme GAMT and creatine kinases uCK-Mi and CK-B suggest a novel neuron-glial relationship for brain energy homeostasis. Eur. J. Neurosci., 20,144-160.

Tamamaki, N., Yanagawa, Y., Tomioka, R., Miyazaki, J.I., Obata, K. \& Kaneko, T. (2003) Green fluorescent protein expression and colocalization with calretinin, parvalbumin, and somatostatin in the GAD67-GFP knock-in mouse, J. Comp. Neurol., 467, 60-79.

Taniike, M., Mohri, I., Eguchi, N., Beuckmann, C.T., Suzuki, K. \& Urade, Y. (2002) Perineuronal oligodendrocytes protect against neuronal apoptosis through the production of lipocalin-type prostaglandin D synthase in a genetic demyelinating model. J. Neurosci., 22, 4885-4896.

Tsacopoulos, M. \& Magistretti, P.J. (1996) Metabolic coupling between glia and neurons. J. Neurosci., 16, 877-885. 
Urade Y, Kitahama K, Ohishi H, Kaneko T, Mizuno N, Hayaishi O (1993) Dominant expression of mRNA for prostaglandin D synthase in leptomeninges, choroid plexus, and oligodendrocytes of the adult rat bran. Proc. Natl. Acad. Sci., USA, 90, 9070-9074.

Vostrikov, V.M., Uranova, N.A. \& Orlovskaya, D.D. (2007) Deficit of perineuronal oligodendrocytes in the prefrontal cortex in schizophrenia and mood disorders. Schizophr. Res., 94, 273-280.

Wyss, M. \& Kaddurah- Daouk, R. (2000) Creatine and creatinine metabolism. Physiol. Rev., 80, 1107-1213.

Yamada, K., Watanabe, M., Shibata, T., Nagashima, M., Tanaka, K. \& Inoue, Y. (1998) Glutamate transporter GLT-1 is transiently localized on growing axons of the mouse spinal cord before establishing astrocytic expression. J. Neurosci., 18, 5706-5713.

Yamada, K., Fukaya, M., Shibata, T., Kurihara, H., Tanaka, K., Inoue, Y. \& Watanabe, M (2000) Dynamic transformation of Bergmann glial fibers proceeds in correlation with dendritic outgrowth and synapse formation of cerebellar Purkinje cells. J. Comp. Neurol., 418, 106-120.

Yamasaki, M., Yamada, K., Furuya, S., Mitoma, J., Hirabayashi, Y. \& Watanabe, M. (2001) 3-Phosphoglycerate dehydrogenase, a key enzyme for L-serine biosynthesis, is preferentially expressed in the radial glia/astrocyte lineage and olfactory ensheathing glia in the mouse brain. J. Neurosci., 21, 7691-7704. 
Yamasaki M, Matsui M, Watanabe M (2010) Preferential localization of muscarinic M1 receptor on dendritic shaft and spine of cortical pyramidal cells and its anatomical evidence for volume transmission. J. Neurosci., 30, 4408-4418.

Yamashita, N., Sakai, K., Furuya, S. \& Watanabe, M. (2003) Selective expression of L-serine synthetic enzyme 3PGDH in schwann cells, perineuronal glia, and endoneurial fibroblasts along rat sciatic nerves and its upregulation after crush injury. Arch, Histol. Cytol., 66, 429-436. 


\section{FIGURE LEGENDS}

Fig. 1. The specificity of antibodies and immunohistochemistry. A. Immunoblot with extracts from the adult mouse cerebral cortex. Primary antibodies raised against CNP, GAMT, Phgdh, GS, and GLAST in the mouse (Ms), rabbit (Rb), or guinea pig (GP) recognize single protein bands at expected protein sizes. The position of standard protein markers is indicated to the left $(\mathrm{kDa})$. B-E, Immunofluorescence in the cerebral cortex with use of rabbit $(B, C)$ and guinea pig (D, E). GS antibodies in the absence (B, D) or presence (C, E) of antigen proteins. Scale bars: B, $100 \mu \mathrm{m}$.

Fig. 2. Specificity of immunohistochemical labeling with use of mouse monoclonal CNP antibody. A-C. Double FISH for CNP (red) and PLP (green) mRNAs. Note almost complete overlap of cellular populations expressing CNP and PLP mRNAs in the adult mouse brain. Distinct cellular expressions are noted in that CNP mRNA, but not PLP mRNA, is expression in Bergmann glia of the cerebellar cortex, whereas PLP mRNA, but not CNP mRNA, is expressed in the olfactory nerve layer of the olfactory bulb. D, E. Triple fluorescent labeling for CNP mRNA (red), CNP protein (green), and nucleus (blue) in the cerebral cortex. Perikaryal labeling of CNP protein completely overlaps with that of CNP mRNA. Arrowhead and arrows indicate CNP-positive perikarya that appear to attach or not to attach, respectively, to putative neurons having large cell bodies. Also note CNP immunolabeling in putative myelin sheath. Cb, cerebellum; cc, corpus callosum; Cx, cortex; Hi, hippocampus; Mb, midbrain; MO, medulla oblongata; OB, olfactory bulb; St, striatum; Th, thalamus; I-VI, cortical layers I through VI. Scale bars, A, 1 mm; B, $200 \mu \mathrm{m}$; C, $10 \mu \mathrm{m} ; \mathrm{D}, 50 \mu \mathrm{m}$; E, $10 \mu \mathrm{m}$.

Fig. 3. Expression of CNP in perineuronal oligodendrocytes and lack of other glial markers in CNP-positive perineuronal oligodendrocytes in the adult somatosensory 
cortex. A. Double fluorescent labeling for CNP and Nissl stain NTG. B. Double immunofluorescence for CNP (green) and neuronal marker MAP2 (blue). C-F. Triple immunofluorescence for CNP (green), MAP2 (blue), and glial markers (red): BLBP for astrocytes (C), GFAP for astrocytes (D), Iba-1 for microglia (E), and NG2 for $\mathrm{NG}^{+}$glia (F). Arrowheads indicate CNP-positive perineuronal oligodendrocytes attaching to MAP2-positive neurons (asterisks). G. FISH for L-PGDS mRNA in the adult mouse cortex. Note that antisense riboprobe for L-PGDS mRNA produces intense signals along the pia mater (PM) and choroid plexus (ChP), and also labels dispersed cells in the cortex (Cx) and corpus callosum (CC). Inset shows blank labeling with use of the sense probe. H. Double FISH for CNP and L-PGDS mRNAs in the somatosensory cortex. CNP mRNA-expressing satellite cells (red) attaching to putative neurons (asterisks) also express L-PGDS mRNA (green). Scale bars, A, $100 \mu \mathrm{m}$; B-F, H, $10 \mu \mathrm{m}$, G, 1mm.H.

Fig. 4. Triple immunofluorescence for glial metabolic enzymes and glutamate transporters in perineuronal oligodendrocyte of the adult mouse cerebral cortex. Sections were immunostained for CNP (green), MAP2 (blue), and glial enzymes and transporters (red): GAMT (A), Phgdh (B), GS (C, D), GLAST (E), and GLT-1 (F). Arrows and arrowheads indicate perineuronal oligodendrocyte expressing or lacking, respectively, glial metabolic enzymes and glutamate transporters. Asterisks indicate neuronal somata attached by perineuronal oligodendrocytes. Scale bars, A, E, G, $10 \mu \mathrm{m}$ : B, D, $1 \mathrm{~mm}$; C, E, $200 \mu \mathrm{m}$.

Fig. 5. Lack of GLT1 and GLAST expression in CNP-expressing perineuronal oligodendrocytes. A. Closer views of triple immunofluorescence CNP (green), GLT1 (red), and MAP2 (blue). Note that GLT1 is negative not only in perikarya (arrowhead), 
but also in processes (arrows) of CNP-positive perineuronal oligodendrocytes attaching to a MAP2-positive neuron (asterisk). B-G. Double FISH for CNP mRNA and GLT1 mRNA (B, C, F) or GLAST mRNA (Fig. 5D, E, G). Note negative hybridizing signals for the two glutamate transporters in CNP mRNA-expressing oligodendrocytes in both the white and gray matters. See abbreviations in Fig. 2. Scale bars, A, F, $10 \mu \mathrm{m} ; \mathrm{B}, 1$ mm; D, $100 \mu \mathrm{m}$.

Fig. 6. Silver-enhanced immunogold electron microscopy for GAMT in the adult cerebral cortex. A. Low-power electron micrograph shows a GAMT-labeled perineuronal oligodendrocyte (PO) tightly contacting the soma of neuron (Neu). B-D. High-power images taken from A. Filled and open arrowheads indicate the cell membrane of perineuronal oligodendrocyte and neuron, respectively. Arrows indicate metal particles for GAMT. Scale bars, A, $1 \mu \mathrm{m}$, B-D, $200 \mathrm{~nm}$.

Fig. 7 Postnatal differentiation of perineuronal oligodendrocytes (A-D) and their preferential attachment to principal cortical neurons (E-H). A-D. GAMT-positive perineuronal oligodendrocytes in the cerebral cortex at P0 (A), P7 (B), P14 (C), and P21 (D). Sections were immunostained for MAP2 (blue), NTG (green), and GAMT (red). Asterisks indicate neuronal somata attached by perineuronal oligodendrocytes (arrows). E-H. Perineuronal oligodendrocytes attach preferentially to glutamatergic neurons. Triple immunofluorescence for CNP (red), MAP2 (blue), and GFP (green) was applied to the cerebral cortex of GAD67-GFP mice at P14 (E, F) and adult stage (G, H). As shown in Panels E and G, GFP-negative glutamatergic neurons (asterisks) are often contacted by perineuronal oligodendrocytes (arrowheads). Panels $\mathrm{F}$ and $\mathrm{H}$ show rare cases in which GFP-positive GABAergic neurons are contacted by perineuronal oligodendrocytes. Scale bars, $10 \mu \mathrm{m}$. 

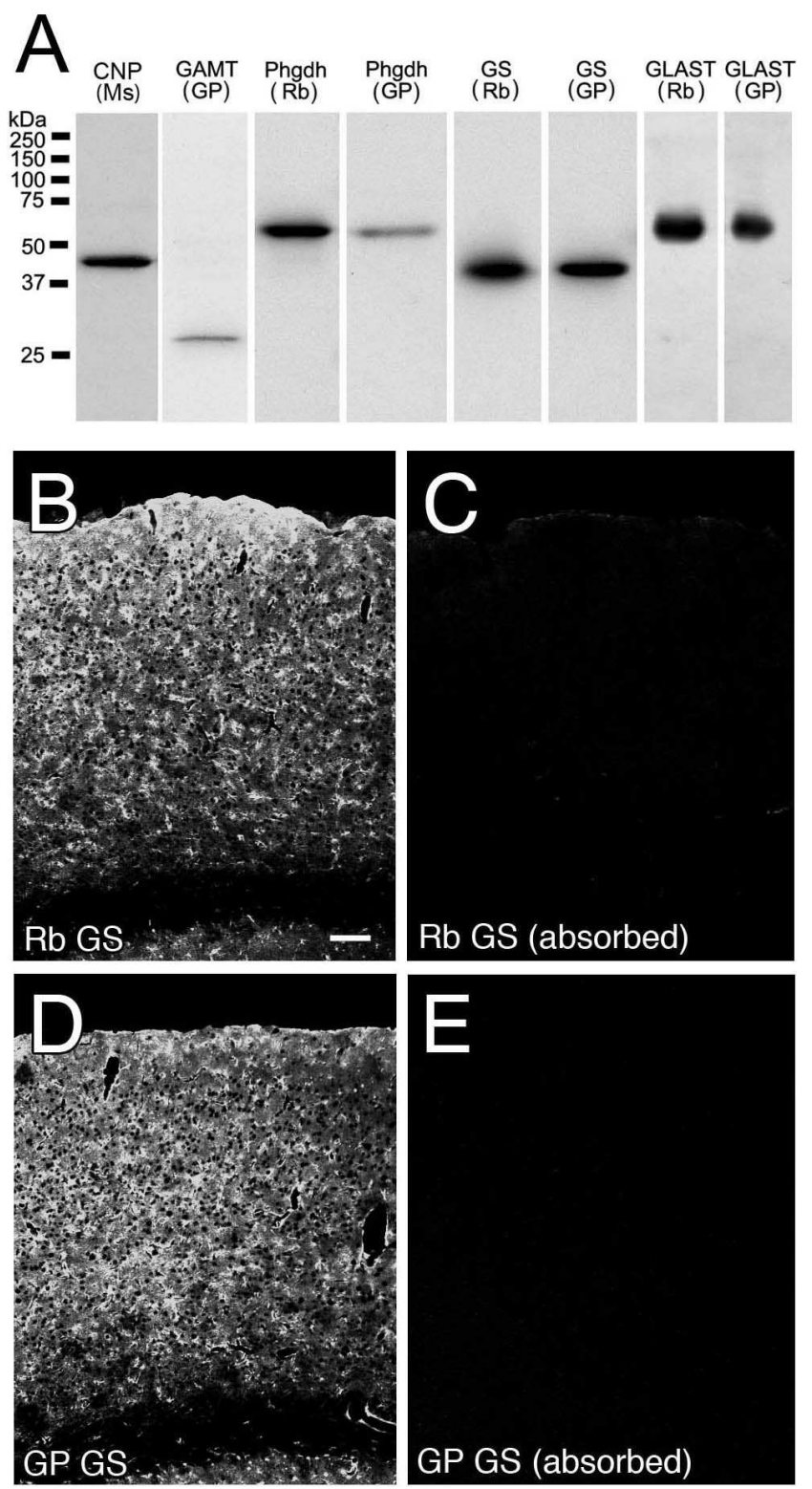

Fig. 1. The specificity of antibodies and immunohistochemistry. A. Immunoblot with extracts from the adult mouse cerebral cortex. Primary antibodies raised against CNP, GAMT, Phgdh, GS, and GLAST in the mouse (Ms), rabbit (Rb), or guinea pig (GP) recognize single protein bands at expected protein sizes. The position of standard protein markers is indicated to the left (kDa). B-E, Immunofluorescence in the cerebral cortex with use of rabbit $(B, C)$ and guinea pig $(D, E)$. GS antibodies in the absence $(B, D)$ or presence $(C, E)$ of antigen proteins. Scale bars: $B, 100 \mathrm{um}$. $83 \times 157 \mathrm{~mm}(300 \times 300 \mathrm{DPI})$ 

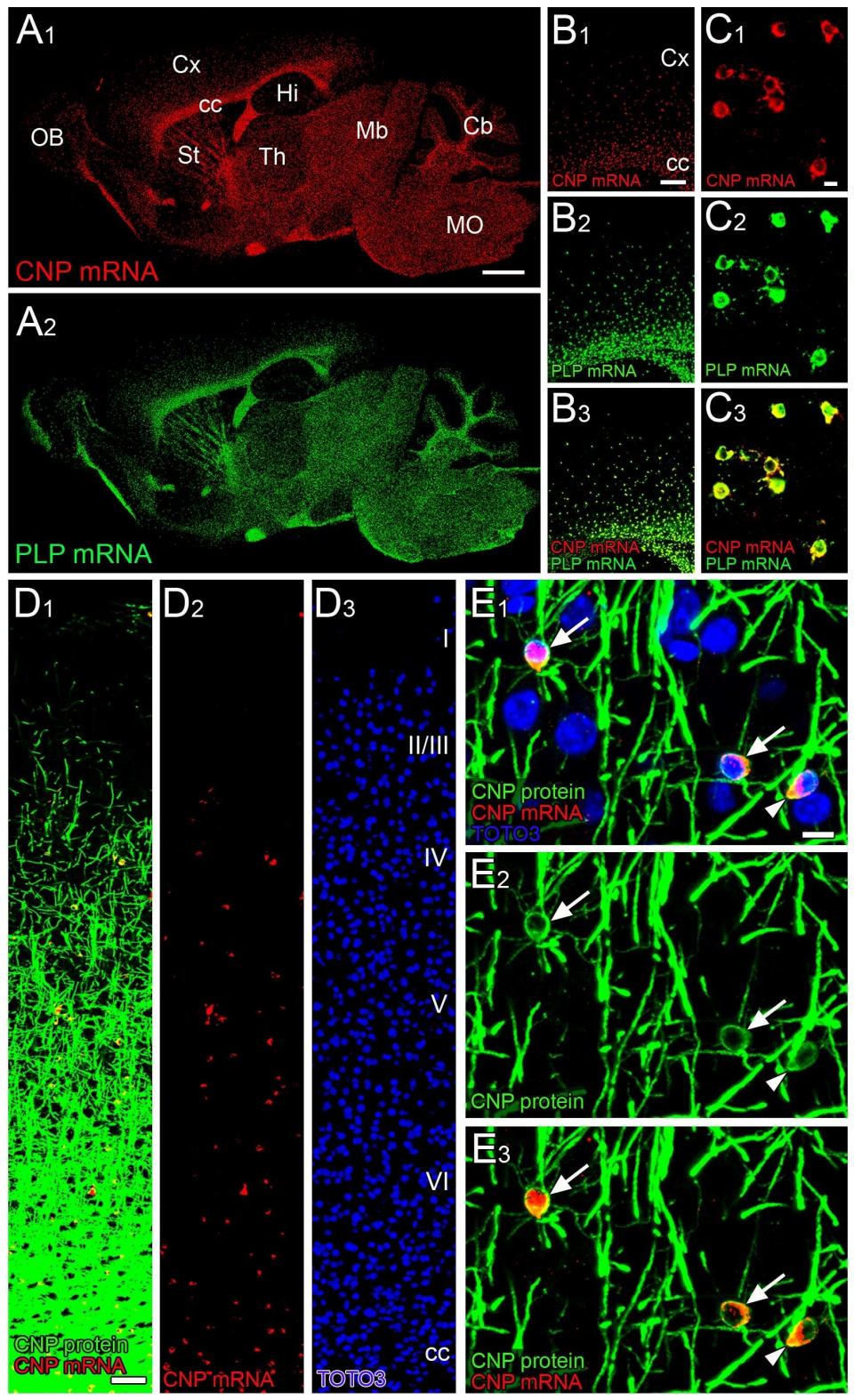

Fig. 2. Specificity of immunohistochemical labeling with use of mouse monoclonal CNP antibody. AC. Double FISH for CNP (red) and PLP (green) mRNAs. Note almost complete overlap of cellular populations expressing CNP and PLP mRNAs in the adult mouse brain. Distinct cellular expressions are noted in that CNP MRNA, but not PLP MRNA, is expression in Bergmann glia of the cerebellar cortex, whereas PLP MRNA, but not CNP mRNA, is expressed in the olfactory nerve layer of the olfactory bulb. D, E. Triple fluorescent labeling for CNP mRNA (red), CNP protein (green), and nucleus (blue) in the cerebral cortex. Perikaryal labeling of CNP protein completely overlaps with that of CNP mRNA. Arrowhead and arrows indicate CNP-positive perikarya that appear to attach or

not to attach, respectively, to putative neurons having large cell bodies. Also note CNP immunolabeling in putative myelin sheath. $\mathrm{Cb}$, cerebellum; $\mathrm{cc}$, corpus callosum; Cx, cortex; $\mathrm{Hi}$,

hippocampus; Mb, midbrain; MO, medulla oblongata; OB, olfactory bulb; St, striatum; Th, thalamus; I-VI, cortical layers I through VI. Scale bars, A, 1 mm; B, 200 um; C, 10 um; D, 50 um; 
E, 10 um.

$142 \times 233 \mathrm{~mm}(300 \times 300 \mathrm{DPI})$ 


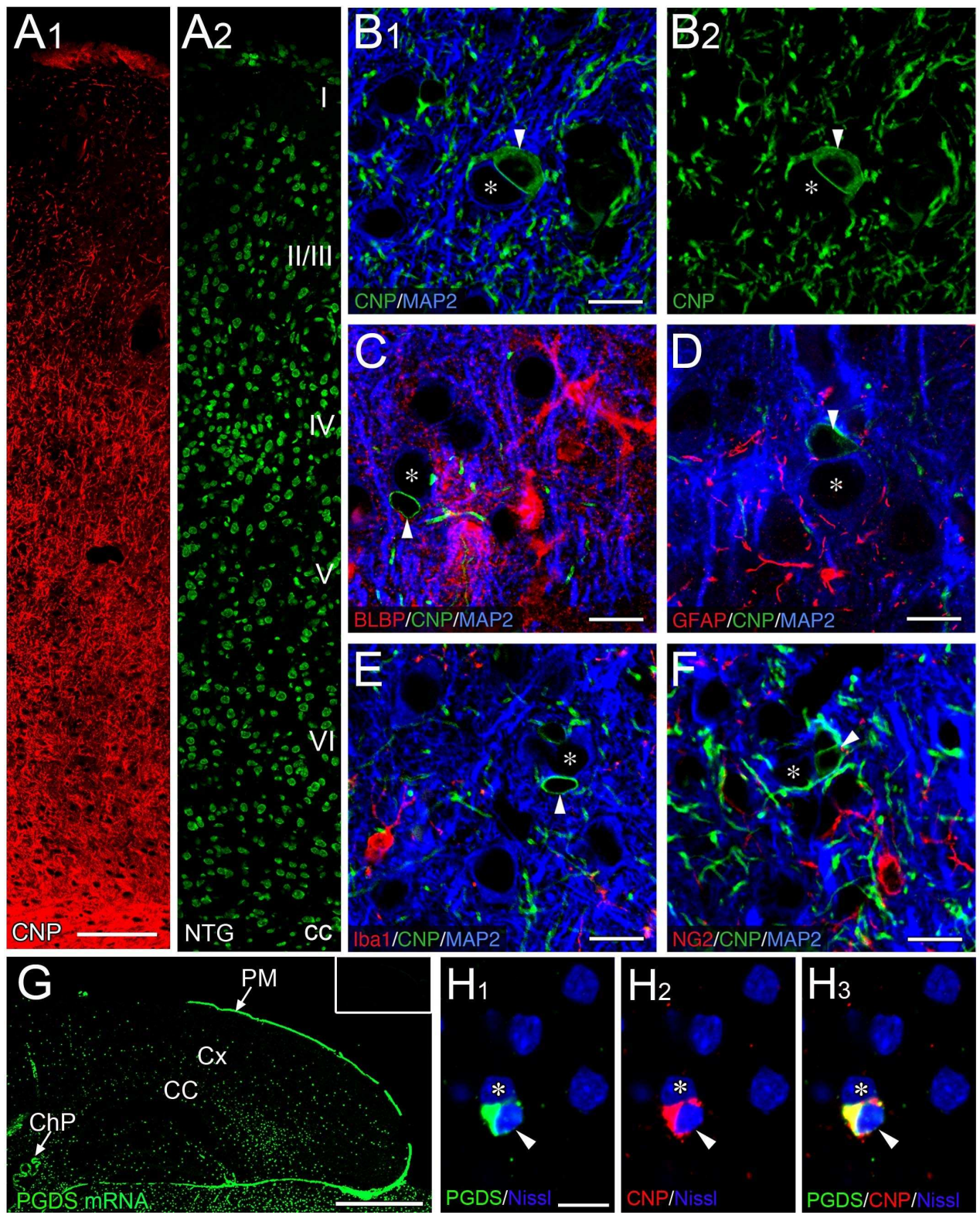

Fig. 3. Expression of CNP in perineuronal oligodendrocytes and lack of other glial markers in CNPpositive perineuronal oligodendrocytes in the adult somatosensory cortex. A. Double fluorescent labeling for CNP and Nissl stain NTG. B. Double immunofluorescence for CNP (green) and neuronal marker MAP2 (blue). C-F. Triple immunofluorescence for CNP (green), MAP2 (blue), and glial markers (red): BLBP for astrocytes (C), GFAP for astrocytes (D), Iba-1 for microglia (E), and NG2 for NG2+ glia (F). Arrowheads indicate CNP-positive perineuronal oligodendrocytes attaching to MAP2-positive neurons (asterisks). G. FISH for L-PGDS mRNA in the adult mouse cortex. Note that antisense riboprobe for L-PGDS mRNA produces intense signals along the pia mater (PM) and choroid plexus (ChP), and also labels dispersed cells in the cortex (Cx) and corpus callosum (CC). Inset shows blank labeling with use of the sense probe. $\mathrm{H}$. Double FISH for CNP and L-PGDS mRNAs in the somatosensory cortex. CNP mRNA-expressing satellite cells (red) attaching to putative neurons (asterisks) also express L-PGDS mRNA (green). Scale bars, A, 100 um; B-F, H, 10 um, G, $1 \mathrm{~mm} . \mathrm{H}$. 
$180 \times 223 \mathrm{~mm}(300 \times 300 \mathrm{DPI})$ 

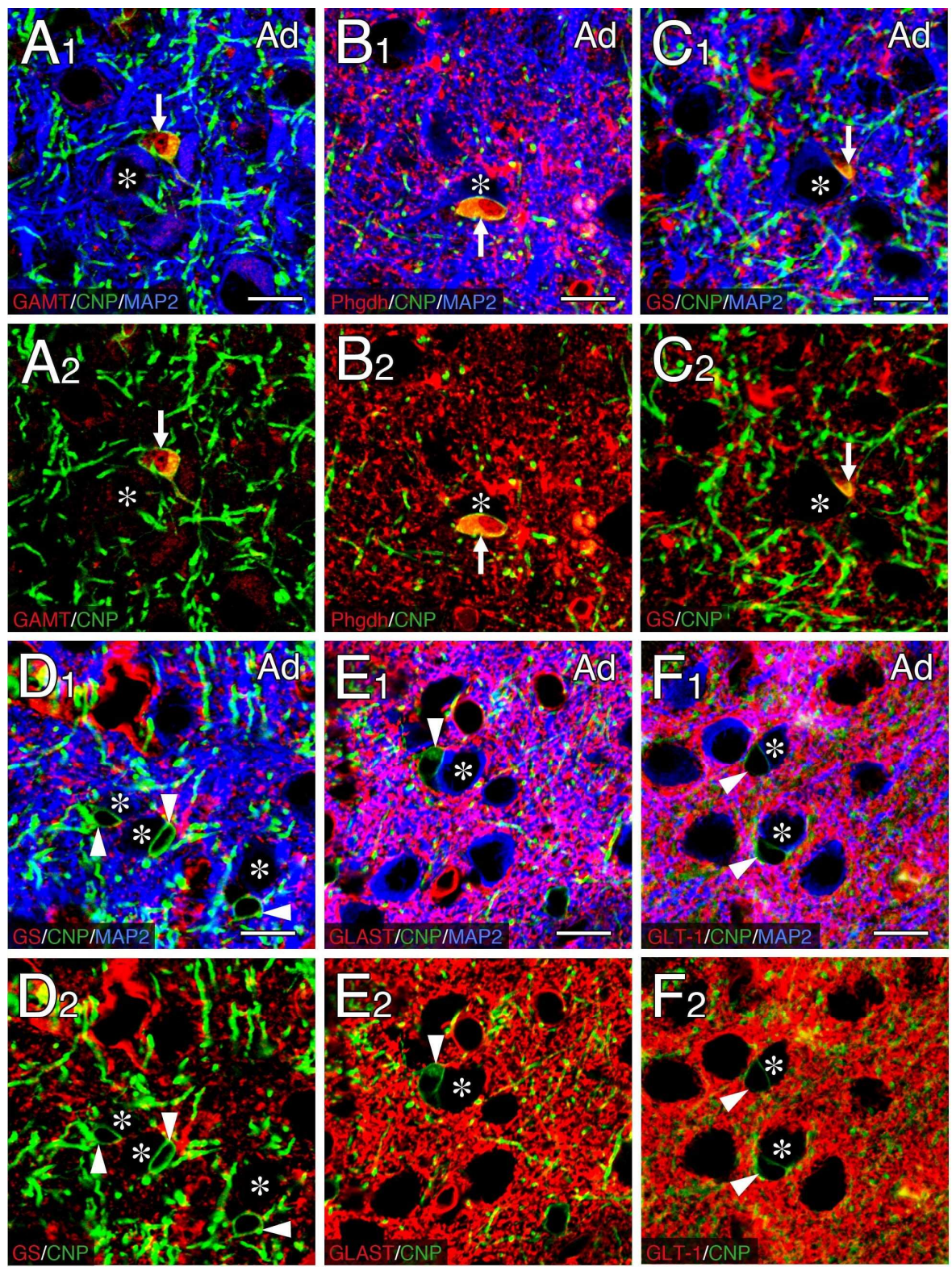

Fig. 4. Triple immunofluorescence for glial metabolic enzymes and glutamate transporters in perineuronal oligodendrocyte of the adult mouse cerebral cortex. Sections were immunostained for CNP (green), MAP2 (blue), and glial enzymes and transporters (red): GAMT (A), Phgdh (B), GS (C,

D), GLAST (E), and GLT-1 (F). Arrows and arrowheads indicate perineuronal oligodendrocyte expressing or lacking, respectively, glial metabolic enzymes and glutamate transporters. Asterisks indicate neuronal somata attached by perineuronal oligodendrocytes. Scale bars, A, E, G, 10 um: B,

D, $1 \mathrm{~mm}$; C, E, 200um.

$166 \times 221 \mathrm{~mm}(300 \times 300 \mathrm{DPI})$ 

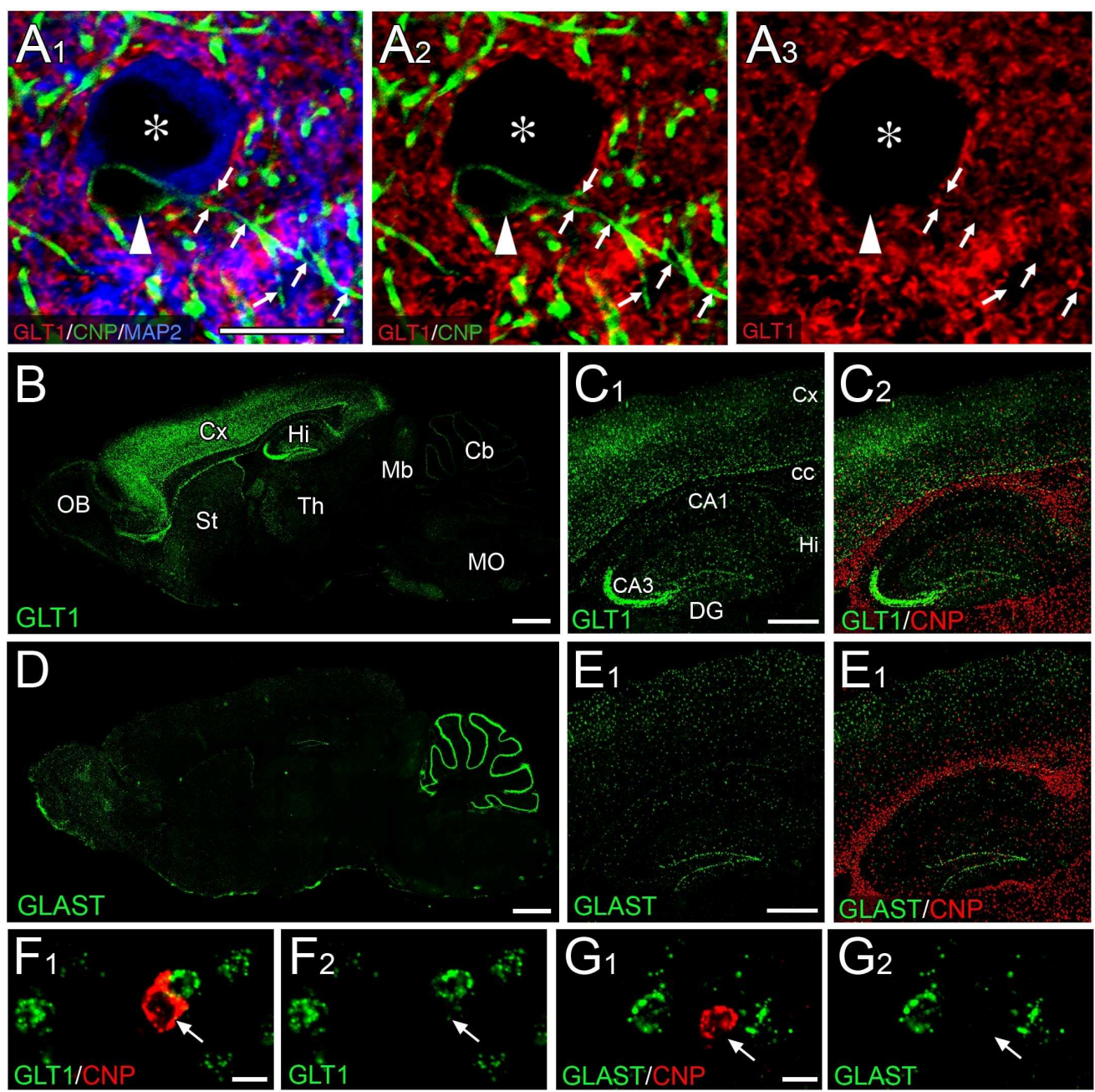

Fig. 5. Lack of GLT1 and GLAST expression in CNP-expressing perineuronal oligodendrocytes. A. Closer views of triple immunofluorescence CNP (green), GLT1 (red), and MAP2 (blue). Note that GLT1 is negative not only in perikarya (arrowhead), but also in processes (arrows) of CNP-positive perineuronal oligodendrocytes attaching to a MAP2-positive neuron (asterisk). B-G. Double FISH for CNP mRNA and GLT1 mRNA (B, C, F) or GLAST mRNA (Fig. 5D, E, G). Note negative hybridizing signals for the two glutamate transporters in CNP mRNA-expressing oligodendrocytes in both the white and gray matters. See abbreviations in Fig. 2. Scale bars, A, F, 10 um; B, 1 mm; D, 100 um. $181 \times 180 \mathrm{~mm}(300 \times 300 \mathrm{DPI})$ 


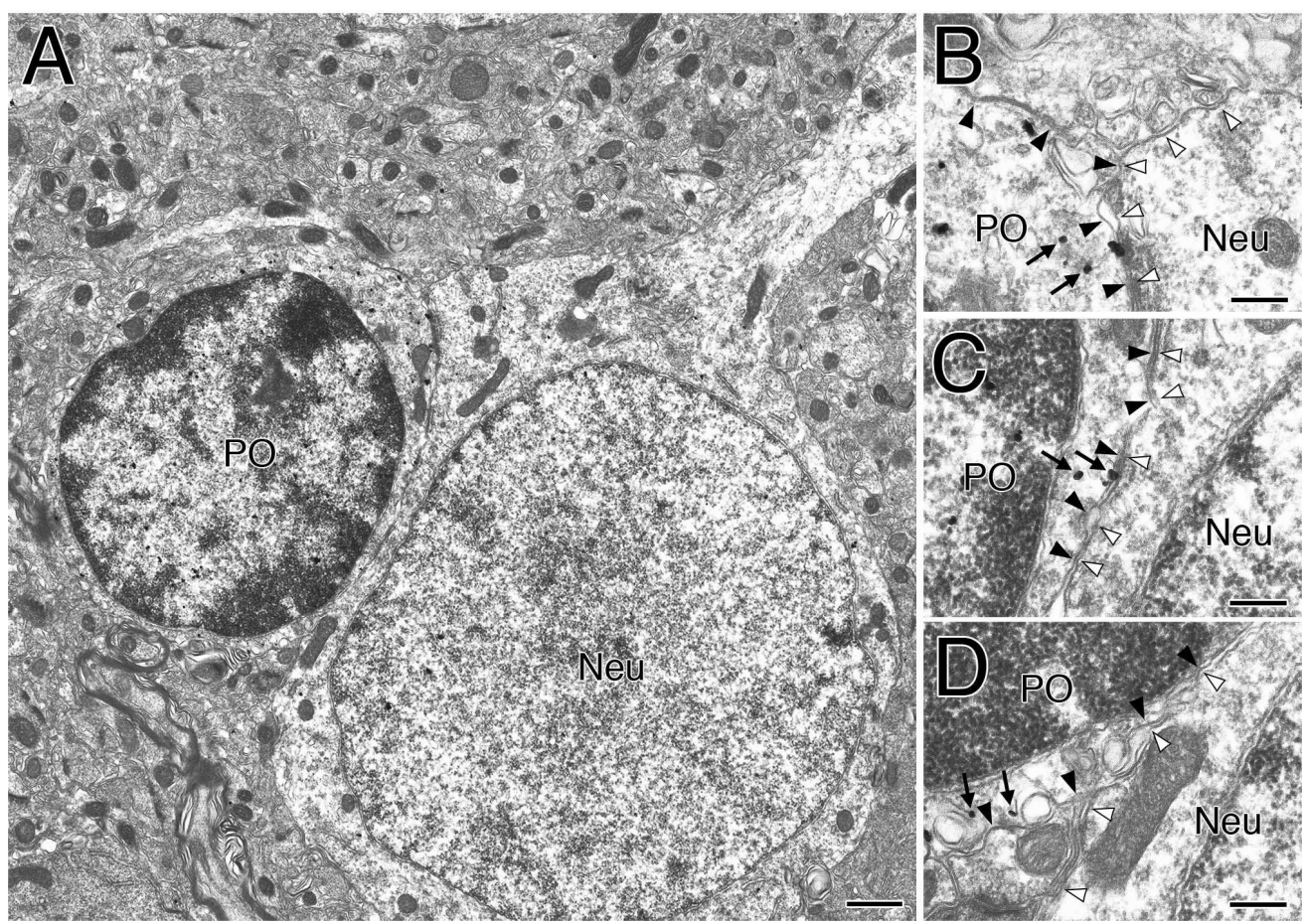

Fig. 6. Silver-enhanced immunogold electron microscopy for GAMT in the adult cerebral cortex. A. Low-power electron micrograph shows a GAMT-labeled perineuronal oligodendrocyte (PO) tightly contacting the soma of neuron (Neu). B-D. High-power images taken from A. Filled and open arrowheads indicate the cell membrane of perineuronal oligodendrocyte and neuron, respectively. Arrows indicate metal particles for GAMT. Scale bars, A, $1 \mathrm{um}, \mathrm{B}-\mathrm{D}, 200 \mathrm{~nm}$. $171 \times 120 \mathrm{~mm}(300 \times 300 \mathrm{DPI})$ 

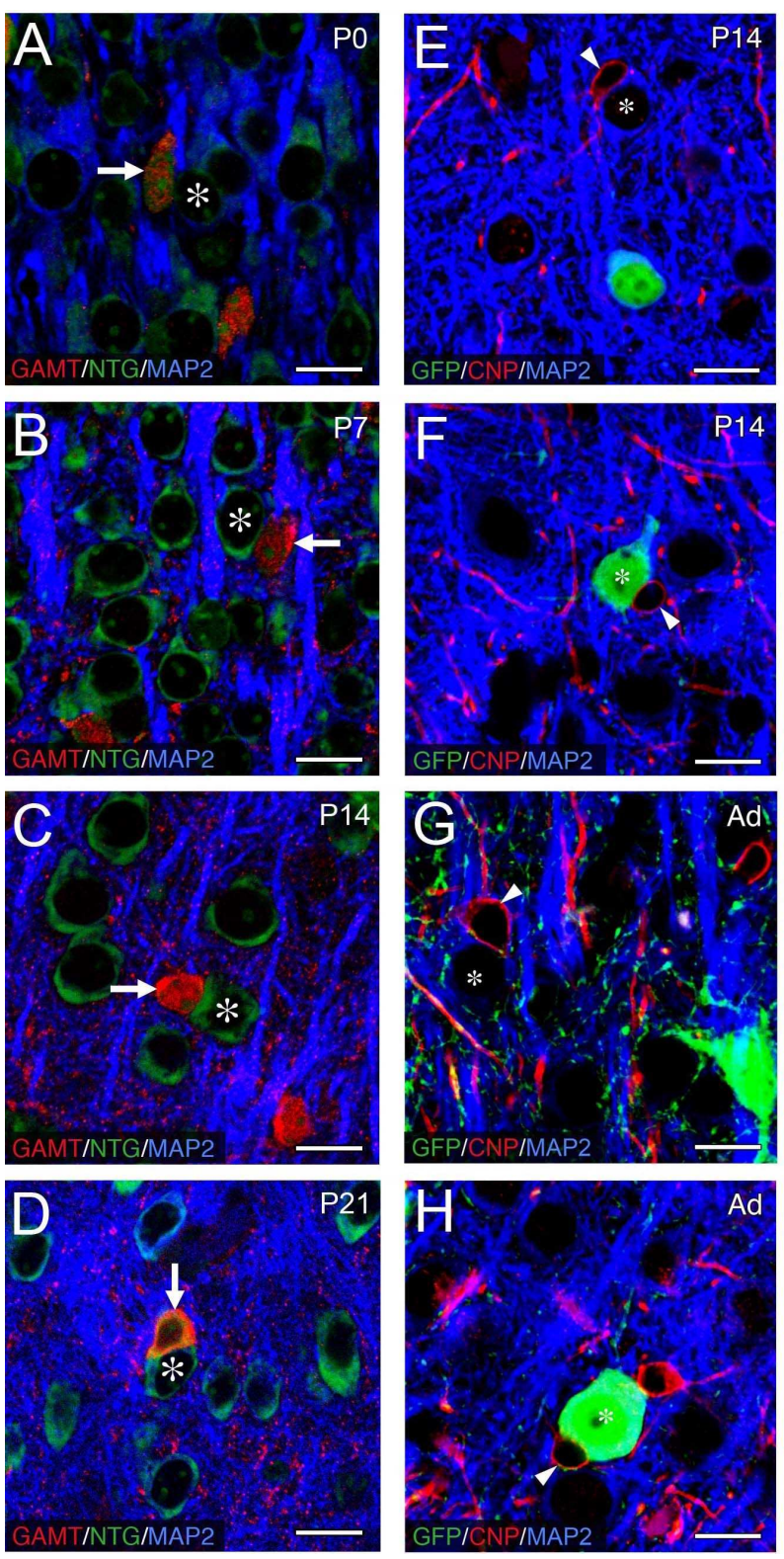

Fig. 7 Postnatal differentiation of perineuronal oligodendrocytes (A-D) and their preferential attachment to principal cortical neurons (E-H). A-D. GAMT-positive perineuronal oligodendrocytes in the cerebral cortex at P0 (A), P7 (B), P14 (C), and P21 (D). Sections were immunostained for MAP2 (blue), NTG (green), and GAMT (red). Asterisks indicate neuronal somata attached by perineuronal oligodendrocytes (arrows). E-H. Perineuronal oligodendrocytes attach preferentially to glutamatergic neurons. Triple immunofluorescence for CNP (red), MAP2 (blue), and GFP (green) was applied to the cerebral cortex of GAD67-GFP mice at P14 (E, F) and adult stage $(G, H)$. As shown in Panels $E$ and

G, GFP-negative glutamatergic neurons (asterisks) are often contacted by perineuronal

oligodendrocytes (arrowheads). Panels $\mathrm{F}$ and $\mathrm{H}$ show rare cases in which GFP-positive GABAergic neurons are contacted by perineuronal oligodendrocytes. Scale bars, 10 um.

$112 \times 222 \mathrm{~mm}(300 \times 300 \mathrm{DPI})$ 


\section{Supporting Information}

Supplemental Fig. S1. Specificity of antibodies to plasmalemmal GABA transporter GAT1. A-F. Double immunofluorescence with antibodies raised against the $N$ - (GAT1-N, rabbit) and $C$-terminal (GAT1-C, guinea pig) sequences of mouse GAT1. Note that almost complete overlap of regional and cellular immunostaining with use of GAT1-N (A, C, D) and GAT1-C (B, E, F) antibodies in the whole brain (A, B), cerebral cortex (C, E), and cerebellar cortex (D, F). G-J. Double immunofluorescence for GAT1 and vesicular inhibitory amino acid transporter (VIAAT). Note that VIAAT (red) accumulates in terminal swellings of GAT1-positive axons (green) in both the cerebral $(\mathrm{G}, \mathrm{H})$ and cerebellar $(\mathrm{H}, \mathrm{J})$ cortices. Also note intense labeling for GAT1 in the pinceau formation, i.e., clustered basket axons surrounding the soma and axon initial segment of Purkinje cells (arrows in I and J). K, L. Immunoblot with GAT1-N (K) and GAT1-C (L) antibodies. GAT1 antibodies raised in the rabbit $(\mathrm{Rb})$ or guinea pig $(\mathrm{GP})$ recognize protein bands at $65 \mathrm{kDa}$. The position of standard protein markers is indicated to the left (kDa). Scale bars, A, B, 1 mm; C-F,15 $\mu \mathrm{m}$; G-J, $5 \mu \mathrm{m}$.

Supplemental Fig. S2. The lack of expression for plasmalemmal GABA transporter GAT1 in CNP-positive perineuronal oligodendrocytes. Triple immunofluorescence CNP (green), GAT1 (red), and MAP2 (blue). Note that GAT1 is negative not only in perikarya (arrowhead), but also in processes (arrows) of CNP-positive perineuronal oligodendrocyte attaching to a MAP2-positive neuron (asterisk). Scale bar, $10 \mu \mathrm{m}$. 

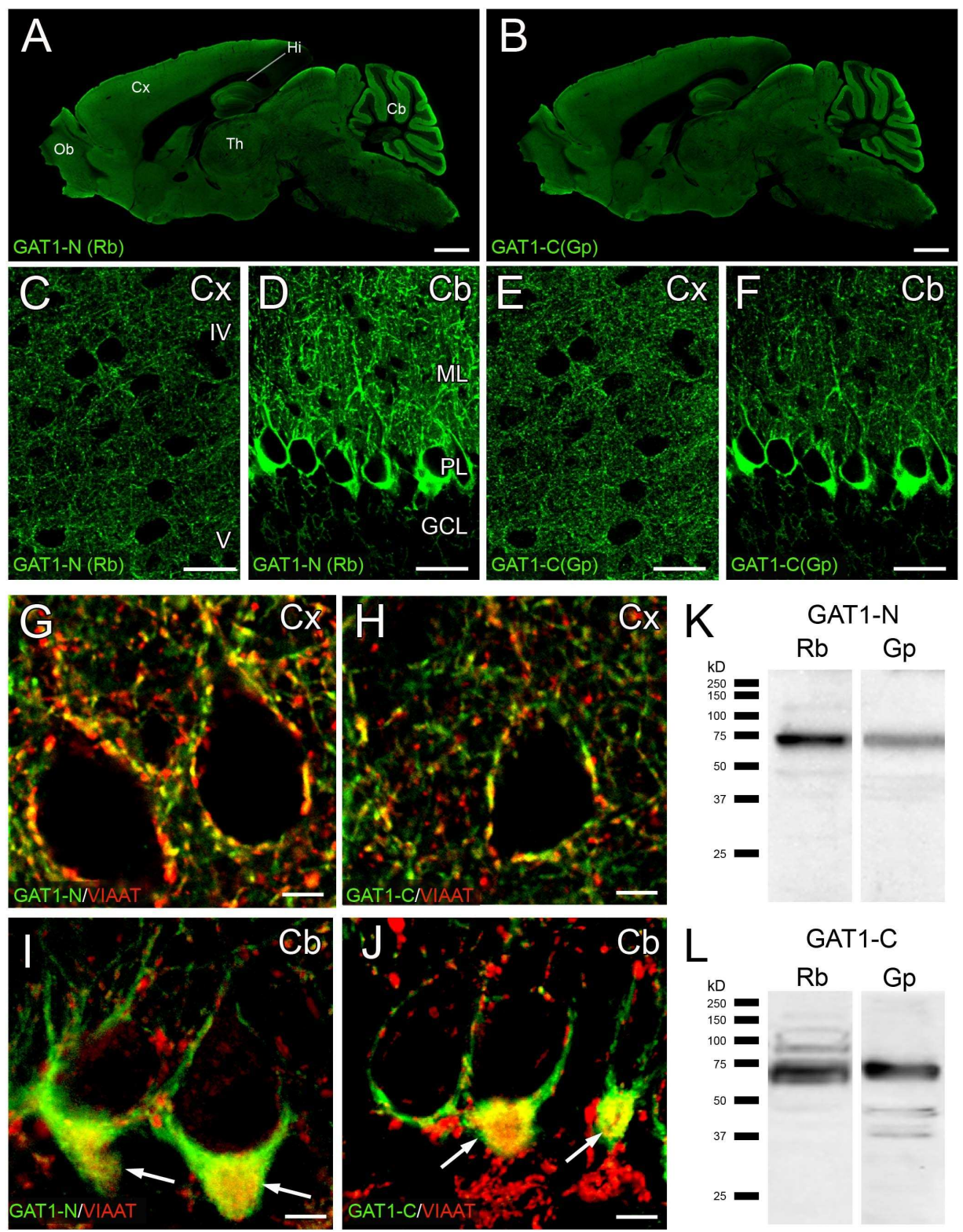

Supplemental Fig. S1. Specificity of antibodies to plasmalemmal GABA transporter GAT1. A-F. Double immunofluorescence with antibodies raised against the $\mathrm{N}$ - (GAT1-N, rabbit) and C-terminal (GAT1-C, guinea pig) sequences of mouse GAT1. Note that almost complete overlap of regional and cellular immunostaining with use of GAT1-N (A, C, D) and GAT1-C (B, E, F) antibodies in the whole brain (A, B), cerebral cortex (C, E), and cerebellar cortex (D, F). G-J. Double immunofluorescence for GAT1 and vesicular inhibitory amino acid transporter (VIAAT). Note that VIAAT (red) accumulates in terminal swellings of GAT1-positive axons (green) in both the cerebral $(\mathrm{G}, \mathrm{H})$ and

cerebellar $(\mathrm{H}, \mathrm{J})$ cortices. Also note intense labeling for GAT1 in the pinceau formation, i.e., clustered basket axons surrounding the soma and axon initial segment of Purkinje cells (arrows in I and J). K, L. Immunoblot with GAT1-N (K) and GAT1-C (L) antibodies. GAT1 antibodies raised in the rabbit $(\mathrm{Rb})$ or guinea pig (GP) recognize protein bands at $65 \mathrm{kDa}$. The position of standard protein markers is indicated to the left (kDa). Scale bars, A, B, $1 \mathrm{~mm}$; C-F, $15 \mathrm{um}$; G-J, $5 \mathrm{um}$.

$175 \times 226 \mathrm{~mm}(300 \times 300 \mathrm{DPI})$ 

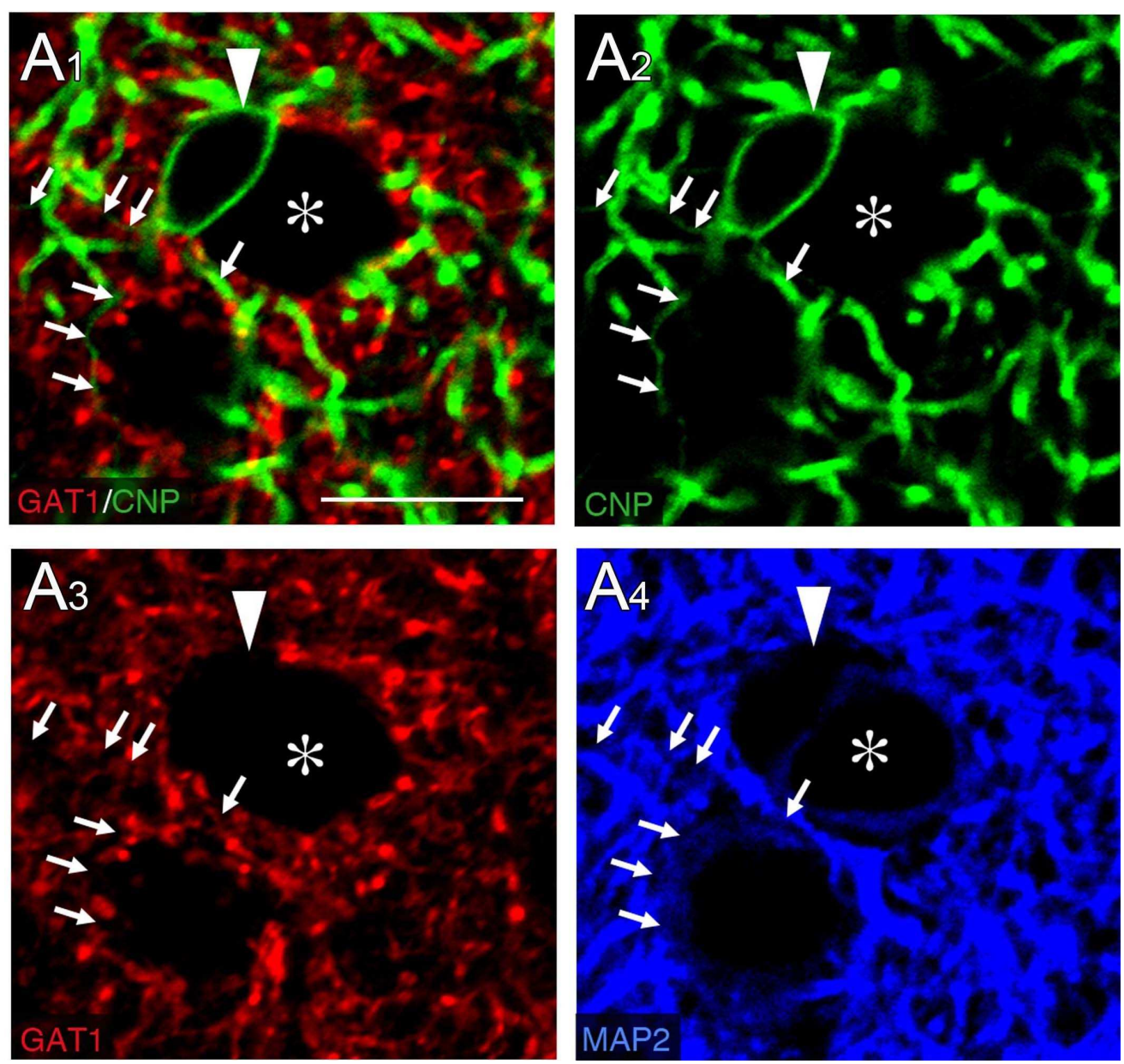

Supplemental Fig. S2. The lack of expression for plasmalemmal GABA transporter GAT1 in CNPpositive perineuronal oligodendrocytes. Triple immunofluorescence CNP (green), GAT1 (red), and MAP2 (blue). Note that GAT1 is negative not only in perikarya (arrowhead), but also in processes

(arrows) of CNP-positive perineuronal oligodendrocyte attaching to a MAP2-positive neuron (asterisk). Scale bar, $10 \mathrm{um}$. $179 \times 168 \mathrm{~mm}(300 \times 300$ DPI $)$ 